\title{
Teaching Video NeuroImages: Wilson Disease Presenting With Catatonia
}

Roshni Abee Patel, MD, and Meagan Bailey, MD, MS

Neurology ${ }^{\circledR}$ 2021;96:e2781-e2782. doi:10.1212/WNL.0000000000012038

A 29-year-old man with a history of depression on fluoxetine presented with 4 months of progressive abdominal pain and hypoactive encephalopathy. On examination, he was nonresponsive, bradykinetic, spastic, and had catatonia with waxy flexibility (video 1 ). Bush-Francis Catatonia Rating Scale score was 28 (table). He had new liver cirrhosis; serum ceruloplasmin was low and 24-hour urine copper was elevated. MRI revealed abnormal signal in basal ganglia and pons (figure). Two heterozygous mutations in the ATP7B gene (c.1934T > G and c.207 G $>\mathrm{T}$ ) confirmed the diagnosis of Wilson disease. Zinc and penicillamine were initiated for copper reduction; catatonia did not respond to benzodiazepines. He was discharged home but required full care from family (nonambulatory, required help with all activities of daily living), and neurologic status was unchanged 3 months after discharge. Catatonia is a rare and underappreciated manifestation in Wilson disease ${ }^{1}$; early recognition is essential for prompt initiation of chelation therapy. ${ }^{2}$

\section{Study Funding}

No targeted funding reported.

\section{Disclosure}

The authors report no disclosures relevant to the manuscript. Go to Neurology.org/N for full disclosures.
Correspondence

Dr. Patel

roshnipatel1988@gmail.com

\section{MORE ONLINE}

$\rightarrow$ Teaching slides

http://links.lww.com/

WNL/B368

- Video 
Table Bush-Frances Catatonia Rating Scale With Subscores

\begin{tabular}{|c|c|}
\hline Signs and symptoms & Patient score $^{a}$ \\
\hline Excitement & 0 \\
\hline Immobility/stupor & 2 \\
\hline Mutism & 3 \\
\hline Staring & 3 \\
\hline Posturing/cataplexy & 3 \\
\hline Grimacing & 3 \\
\hline Echopraxia/echolalia & 0 \\
\hline Stereotypy & 0 \\
\hline Mannerisms & 0 \\
\hline Verbigeration & 1 \\
\hline Rigidity & 2 \\
\hline Negativism & 0 \\
\hline Waxy flexibility & 3 \\
\hline Withdrawal & 2 \\
\hline Impulsivity & 0 \\
\hline Automatic obedience & 1 \\
\hline Mitgehen & 0 \\
\hline Gegenhalten & 0 \\
\hline Ambitendency & 3 \\
\hline Grasp reflex & 0 \\
\hline Perseveration & 3 \\
\hline Combativeness & 1 \\
\hline Autonomic abnormality & 1 \\
\hline Total score & 28 \\
\hline
\end{tabular}

${ }^{a}$ All items are rated on scale of $0-3$
Figure MRI Fluid-Attenuated Inversion Recovery
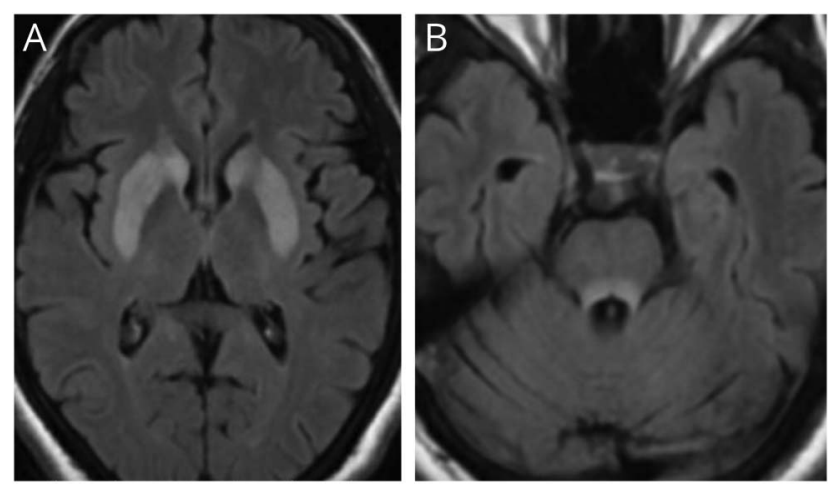

Hyperintensities seen in bilateral caudate and lentiform nuclei (A) and dorsal pons (B).

\section{Appendix Authors}

\begin{tabular}{lll}
\hline Name & Location & Contribution \\
\hline $\begin{array}{l}\text { Roshni } \\
\text { Patel, MD }\end{array}$ & $\begin{array}{l}\text { Rush University } \\
\text { Medical Center, } \\
\text { Chicago, IL }\end{array}$ & $\begin{array}{l}\text { Designed and conceptualized } \\
\text { study, major role in acquisition of } \\
\text { data, drafted the manuscript for } \\
\text { intellectual content }\end{array}$ \\
$\begin{array}{l}\text { Meagan } \\
\text { Bailey, MD, } \\
\text { MS }\end{array}$ & $\begin{array}{l}\text { Rush University } \\
\text { Medical Center, } \\
\text { Chicago, IL }\end{array}$ & $\begin{array}{l}\text { Revised the manuscript for } \\
\text { intellectual content }\end{array}$ \\
\hline
\end{tabular}

\section{References}

1. Basu A, Thanapal S, Sood M, Khandelwal SK. Catatonia: an unusual manifestation of Wilson's disease. J Neuropsychiatry Clin Neurosci. 2015;27:72-73.

2. Bandmann O, Weiss KH, Kaler SG. Wilson's disease and other neurological copper disorders. Lancet Neurol. 2015;14:103-113. 


\title{
Neurology
}

\author{
Teaching Video NeuroImages: Wilson Disease Presenting With Catatonia \\ Roshni Abee Patel and Meagan Bailey \\ Neurology 2021;96;e2781-e2782 Published Online before print April 14, 2021 \\ DOI 10.1212/WNL.0000000000012038
}

This information is current as of April 14, 2021

\section{Updated Information \& Services}

\section{References}

Subspecialty Collections

Permissions \& Licensing

Reprints including high resolution figures, can be found at: http://n.neurology.org/content/96/22/e2781.full

This article cites 2 articles, 0 of which you can access for free at: http://n.neurology.org/content/96/22/e2781.full\#ref-list-1

This article, along with others on similar topics, appears in the following collection(s):

\section{All Genetics}

http://n.neurology.org/cgi/collection/all_genetics

All Psychiatric disorders

http://n.neurology.org/cgi/collection/all_psychiatric_disorders

Metabolic disease (inherited)

http://n.neurology.org/cgi/collection/metabolic_disease_inherited MRI

http://n.neurology.org/cgi/collection/mri

Parkinson's disease/Parkinsonism

http://n.neurology.org/cgi/collection/parkinsons_disease_parkinsonism

Information about reproducing this article in parts (figures,tables) or in its entirety can be found online at:

http://www.neurology.org/about/about_the_journal\#permissions

Information about ordering reprints can be found online:

http://n.neurology.org/subscribers/advertise

Neurology ${ }^{\circledR}$ is the official journal of the American Academy of Neurology. Published continuously since 1951, it is now a weekly with 48 issues per year. Copyright (O) 2021 American Academy of Neurology. All rights reserved. Print ISSN: 0028-3878. Online ISSN: 1526-632X.

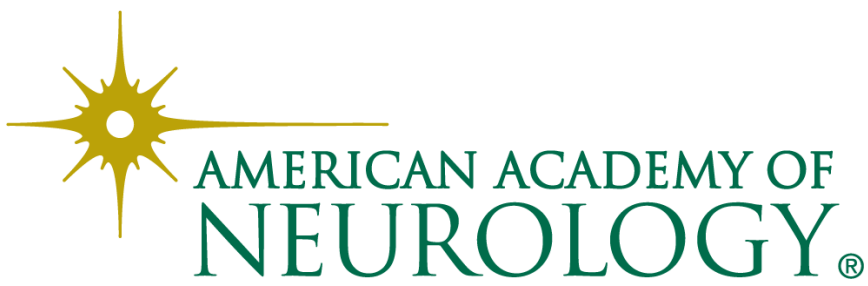

